\section{Parameter sensitivity analysis of the theoretical model of a CR-39-based direct ${ }^{222} \mathrm{Rn} /{ }^{220} \mathrm{Rn}$ progeny monitor}

Jun Hu (D,

Masahiro Hosoda, Shinji Tokonami

\begin{abstract}
The deposition-based direct indoor ${ }^{222} \mathrm{Rn}$ and ${ }^{220} \mathrm{Rn}$ progeny measurement techniques are mostly affected by the indoor environmental conditions, such as the ventilation, concentration of condensation nuclei, and reactions with the structure and its furnishings. In this study, a theoretical model of a direct ${ }^{222} \mathrm{Rn}$ and ${ }^{220} \mathrm{Rn}$ progeny monitor based on allyl diglycol carbonate (ADC or CR-39) was established to analyse the factors that influence the detection process by using the parameter sensitivity analysis. The aerosol parameters contributed the highest to the variance, followed by the aerodynamic parameters. With respect to the result of the Spearman's correlation analysis, the aerosol-related and the room-related parameters are positive, whereas the aerodynamic parameters - which affect the turbulence of indoor deposition - are negative. It means that both the attachment process and the deposition process of ${ }^{222} \mathrm{Rn}$ and ${ }^{220} \mathrm{Rn}$ progenies are important to the performance of the progeny monitor.
\end{abstract}

Keywords: ${ }^{222} \mathrm{Rn}$ and ${ }^{220} \mathrm{Rn}$ progeny $\bullet$ Parameter sensitivity analysis • Theoretical model

\section{J. Hu}

Department of Radiation Sciences

Graduate School of Health Sciences

Hirosaki University

66-1 Hon-cho, Hirosaki, Aomori 036-8564, Japan and Japan Society for the Promotion of Science

Tokyo, Japan

\section{Hosoda}

Department of Radiation Sciences

Graduate School of Health Sciences

Hirosaki University

66-1 Hon-cho, Hirosaki, Aomori 036-8564, Japan and Institute of Radiation Emergency Medicine Hirosaki University

66-1 Honcho, Hirosaki, Aomori 036-8564, Japan

\section{S. Tokonami ${ }^{凶}$}

Institute of Radiation Emergency Medicine

Hirosaki University

66-1 Hon-cho, Hirosaki, Aomori 036-8564, Japan

E-mail: tokonami@hirosaki-u.ac.jp

Received: 27 November 2019

Accepted: 3 February 2020

\section{Introduction}

The evaluation of internal exposure to ${ }^{222} \mathrm{Rn}$ and ${ }^{220} \mathrm{Rn}$ should rely on the equilibrium equivalent concentration (EEC), rather than their concentrations in the air, because the majority of the dose is built up by their progenies. The EEC is specified by the concentrations of ${ }^{222} \mathrm{Rn}$ and ${ }^{220} \mathrm{Rn}$ progenies and depends on their half-lives, indoor ventilation, and deposition on the surface of all kinds of objects, e.g., furniture, floor, ceiling, and wall. The half-life of ${ }^{220} \mathrm{Rn}(55.6 \mathrm{~s})$ is relatively very short. Hence, it is not homogeneously distributed, and its concentration decreases with increasing distance from the source. For all these reasons, a direct technique for the measurement of ${ }^{222} \mathrm{Rn}$ and ${ }^{220} \mathrm{Rn}$ progeny concentration is necessary for the correct assessment of the internal exposure due to these radionuclides.

Recently, direct progeny measurement techniques for the EEC of ${ }^{222} \mathrm{Rn}$ and ${ }^{220} \mathrm{Rn}$ were developed by Tokonami et al. [1] and Mishra and Mayya [2]. The application of the direct measurement technique for determining their progeny concentrations depends on the estimation of the effective deposition velocities of combinations of ${ }^{222} \mathrm{Rn}$ and ${ }^{220} \mathrm{Rn}$ progenies in the typical indoor environment. Some researchers $[2,3]$ have used the experimental method to estimate the geometric mean deposition velocity and applied the data to the actual measurement in the indoor environment. However, because of 


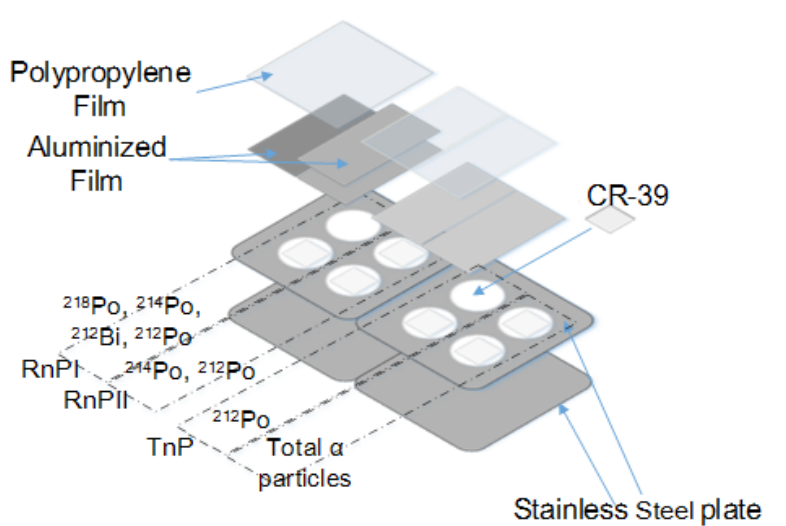

Fig. 1. The schematic diagram of the CR-39-based direct ${ }^{222} \mathrm{Rn} /{ }^{220} \mathrm{Rn}$ progeny monitor.

the fluctuation in environmental factors, the field measurement limitations often do not allow the sufficiently precise estimation of the particle deposition velocities, and as a result, the uncertainty in dose assessment increases. Therefore, in this study, a theoretical model based on the measured value of the environmental parameters has been established to evaluate the factors that influence the direct ${ }^{222} \mathrm{Rn}$ and ${ }^{220} \mathrm{Rn}$ progeny measurements.

\section{Materials and methods}

The direct ${ }^{222} \mathrm{Rn}$ and ${ }^{220} \mathrm{Rn}$ progeny monitor is made up of a stainless steel plate, allyl diglycol carbonate (ADC or CR-39) nuclear track detector, and aluminium-vapourized Mylar film (Fig. 1). Three thicknesses of the films, viz., $7.10 \mathrm{mg} \cdot \mathrm{cm}^{-2}$, $5.05 \mathrm{mg} \cdot \mathrm{cm}^{-2}$, and $3.25 \mathrm{mg} \cdot \mathrm{cm}^{-2}$, are selected to allow for the responding alpha energy to be higher than that emitted by the progeny ${ }^{212} \mathrm{Po},{ }^{214} \mathrm{Po}$, and ${ }^{218} \mathrm{Po}$ to form the tracks on CR-39, respectively. In the behaviour of ${ }^{222} \mathrm{Rn}$ and ${ }^{220} \mathrm{Rn}$ progenies, the relationship between the attached ${ }^{222} \mathrm{Rn}$ and ${ }^{220} \mathrm{Rn}$ progeny deposition rate, $J$ (atoms per square centimeter per second), and the track density, $N$ (tracks per square centimeter), can be expressed as follows [3]:

$$
J=\frac{N}{\eta t}
$$

where $\eta$ is the track registration efficiency, which is the multiplier of the branching ratio of its progeny and the geometric efficiency. For each channel, the geometric efficiency depends on the energies of the incident $\alpha$-particle, the incident angles against the absorbers, and the thickness of the absorbers. And $t$ is the exposure period (in seconds).

The effective deposition velocity of the progeny, $V_{e}$ (meters per second), is defined as follows:

$$
V_{e}=\frac{J}{C}
$$

where $C$ is the atom concentration of the progeny (atoms per cubic meter).

The effective deposition velocity combines the contributions from both the unattached and the attached fractions of each progeny species. By considering the contributions of the two fractions, the deposition velocity of each radionuclide can be written as follows:

$$
V_{i}=p_{i} \cdot V_{d}^{u}+\left(1-p_{i}\right) \cdot V_{d}^{a}
$$

where $p_{i}$ denotes the unattached fraction of radionuclide $i ; V_{d}{ }^{u}$ and $V_{d}{ }^{a}$ are the deposition velocities of the radioactive particles that belong to the unattached and attached fractions, respectively, and are dependent on the aerodynamic factors.

In the theoretical model, the particle deposition velocity is one of the most important parameters in the calculation process. Lai and Nazaroff's three-layer model [4] is a commonly used method to simulate particle deposition. The deposition velocity for the attached fraction can be written as follows:

$$
\begin{gathered}
v_{d, u}=\frac{u^{*}}{1-\exp \left(-\frac{v_{S} I}{u^{*}}\right)} \\
v_{d, d}=\frac{u^{*}}{\exp \left(-\frac{v_{S} I}{u^{*}}\right)-1} \\
v_{d . v}=\frac{u^{*}}{I}
\end{gathered}
$$

where

$$
\begin{aligned}
& I=\left[3.64 S c^{2 / 3}(a-b)+39\right] \\
& a=\frac{1}{2} \operatorname{In}\left[\frac{\left(10.92 S c^{-1 / s}+4.3\right)^{3}}{S c^{-1}+0.0609}\right]+\sqrt{3} \tan ^{-1}\left[\frac{8.6-10.92 S c^{-1 / s}}{\sqrt{3} \cdot 10.92 S c^{-1 / s}}\right] \\
& b=\frac{1}{2} \operatorname{In}\left[\frac{\left(10.92 S c^{-1 / 3}+4.3\right)^{3}}{S c^{-1}+7.669 \times 10^{-4}\left(r^{+}\right)^{3}}\right]+\sqrt{3} \tan ^{-1}\left[\frac{2 r^{+}-10.92 S c^{-1 / 3}}{\sqrt{3} \cdot 10.92 S c^{-1 / 3}}\right] \\
& r^{+}=d_{p} u^{*} /(2 v)
\end{aligned}
$$

In the above formulas, the terms $v_{d, u}, v_{d, d}$, and $v_{d, v}$ are the deposition velocities of the upward horizontal surface, downward horizontal surface, and vertical surface, respectively; $S c=v / D$, where $v$ is the kinematic viscosity of air; and $D$ is the Brownian diffusivity of the particle. Moreover, $d_{p}$ is the particle diameter; $u^{*}$ is the friction velocity; and $v_{S}$ is the gravitational settling velocity of the particle.

After resolving the deposition velocity of the radionuclides, the mass balance-based Jacobi room model [5] is adopted to deduce the concentrations of ${ }^{222} \mathrm{Rn}$ and ${ }^{220} \mathrm{Rn}$ progenies and estimate the EEC. In order to establish the Jacobi room model, the composition of the deposited progeny atoms should be known. The deposited progeny atoms ${ }^{212} \mathrm{Bi}$ and ${ }^{212} \mathrm{~Pb}$ decay ultimately to ${ }^{212}$ Po and form tracks on CR-39. Accordingly, ${ }^{218} \mathrm{Po},{ }^{214} \mathrm{Bi}$, and ${ }^{214} \mathrm{~Pb}$ are deposited on the surface of the monitor and decay to ${ }^{214} \mathrm{Po}$, forming tracks on CR-39.

Since this progeny monitor is very sensitive to environmental conditions, in this study, we use the Monte Carlo sampling method to analyse the contribution of the typical parameters to the EEC of each 
Table 1. The parameters of the sensitivity analysis

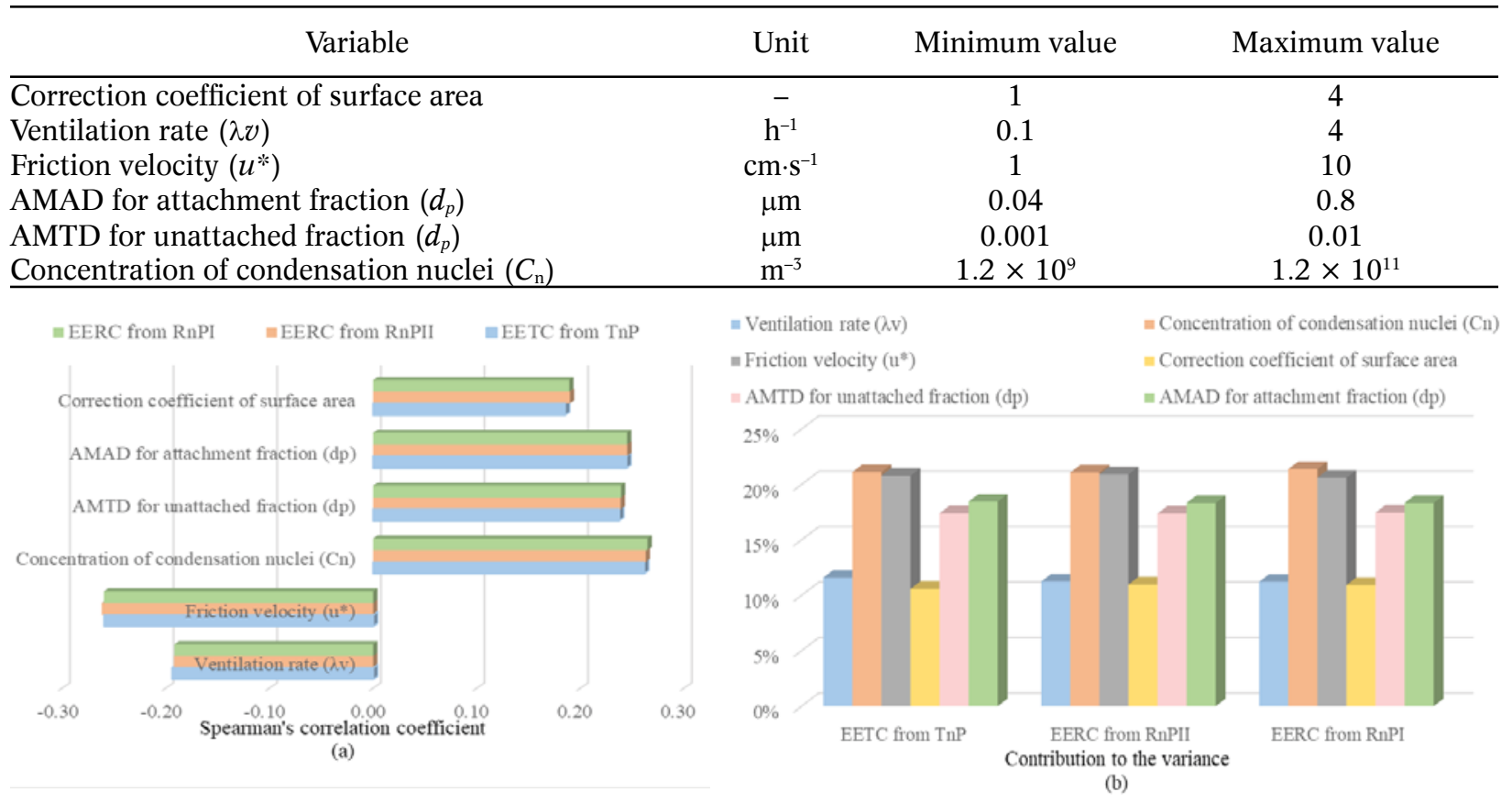

Fig. 2. The results of parameter sensitivity analysis.

detecting channel and, moreover, analyse the factors that influence the performance of the monitor.

\section{Results}

In this theoretical model, we set the aerosol particle parameters, aerodynamic parameters, and room-related parameter as the main parameters to carry out the parameter sensitivity analysis based on the behaviour of the indoor ${ }^{222} \mathrm{Rn}$ and ${ }^{220} \mathrm{Rn}$ progenies. The aerosol particle parameters include the activity median aerodynamic diameter (AMAD) for the attachment fraction, the activity median thermodynamic diameter (AMTD) for the unattached fraction, and the concentration of the condensation nuclei, which indicates the aerosol distribution and concentration in the indoor environment. The aerodynamic parameters for sensitivity analysis selected in this study are the friction velocity and the ventilation rate. Both of the aerosol parameters and the aerodynamic parameters are the main parameters used to estimate the deposition velocity of the progeny radionuclide on the plate surface. The room-related parameter in the theoretical model is the correction coefficient of the surface area, which decides the ratio of the progeny radionuclide deposed on the limited area of the surface of the plate detector. Subsequently, the parameter sensitivity analysis based on the Monte Carlo method was implemented to analyse the contribution of the parameters to the values of the equilibrium equivalent radon concentration (EERC) and the equilibrium equivalent thoron concentration (EETC) for each detecting channel. We set the uniform distribution to the range of $1-10$ tracks. $\mathrm{mm}^{-2}$ for the equal contribution of each progeny radionuclide. Then, 100000 samples from the uniform distributions of the range (Table 1) for each parameter were randomly extracted to run the theoretical model. Subsequently, the Spearman's correlation coefficients and their contribution to the variance were calculated as shown in Fig. 2.

As a result, the friction velocity and ventilation rate for all the channels were negative correlated with the EEC. The other parameters had positive correlations. With respect to the contribution to variance, the aerosol particle parameters had the highest contribution, followed by the aerodynamic parameters and room-related parameter. According to the separate parameters for each channel, six parameters had the same ratio of contribution to each channel. The concentration of the condensation nuclei had the highest contribution, with an average around $21.21 \%$, followed by the friction velocity, which was around $20.79 \%$. The other two parameters of the aerosol particle, viz., the AMAD for the attached and AMTD for the unattached fractions, also contributed relatively greatly to the variance, which were $18.37 \%$ and $17.42 \%$, respectively.

\section{Conclusions}

In this research, a theoretical model was applied to simulate the mechanism of deposition based on ${ }^{222} \mathrm{Rn}$ and ${ }^{220} \mathrm{Rn}$ progeny measurements. Sensitivity analysis of the parameters was carried out to analyse the factors that influence the monitor. Since the attachment process is a function of particle size and the deposition velocity is mostly dependent on the aerodynamic condition, according to the results of the sensitivity analysis, it is indicated that both the deposition and the attachment processes are important to the performance of the direct ${ }^{222} \mathrm{Rn}$ and ${ }^{220} \mathrm{Rn}$ progeny measurement technique. In the 
actual measurement, it is recommended to use the in-site measurement data of the aerosol parameters to calibrate the monitor.

Acknowledgments. This work was supported by the Japan Society for the Promotion of Science (JSPS) KAKENHI vide grant numbers JP19J14291 and partially supported by grants JP16K15368 and JP16H02667.

\section{ORCID}

J. Hu (D) http://orcid.org/0000-0002-9974-6958

\section{References}

1. Tokonami, S., Sun, Q., Yonehara, H., \& Yamada, Y. (2002). A simple measurement technique of equilibrium equivalent thoron concentration with a CR-39 detector. Jpn. J. Health Phys., 37(1), 59-63. DOI: 10.5453/jhps.37.59.

2. Mishra, R., \& Mayya, Y. S. (2008). Study of a deposition-based direct thoron progeny sensor (DTPS) technique for estimating equilibrium equivalent thoron concentration (EETC) in indoor environment. Radiat. Meas., 43(8), 1408-1416. DOI: 10.1016/j. radmeas.2008.03.002.

3. Zhuo, W., \& Iida, T. (2000). Estimation of thoron progeny concentration in dwellings with their deposition rate measurements. Jpn. J. Health Phys., 35(3), 365-370. DOI: 10.5453/jhps.35.365.

4. Lai, C. K., \& Nazarff, W. (2000). Modeling indoor particle deposition from turbulent flow onto smooth surfaces. J. Aerosol Sci., 31(4), 463-476. DOI: 10.1016/S0021-8502(99)00536-4.

5. Jacobi, W. (1972). Activity and potential $\alpha$-energy of ${ }^{222} \mathrm{Rn}$ and ${ }^{220} \mathrm{Rn}$-daughters in different air atmospheres. Health Phys., 22(5), 441-450. DOI: 10.1097/00004032-197205000-00002. 